\title{
Mechanisms of self-assembly and fibrillization of the prion-like domains
}

\author{
Yimei Lu†, Liangzhong Lim†, Yanming Tan†, Lu Wang and Jianxing Song* \\ Department of Biological Sciences, Faculty of Science, National University of Singapore; 10 Kent \\ Ridge Crescent, Singapore 119260;
}

Short title: Self-assembly and fibrillization of the prion-like domains

$\dagger$ The first three authors contribute equally.

*: corresponding author; $\underline{\text { dbssjx @ nus.edu.sg. }}$

Key words: Amyotrophic lateral sclerosis (ALS); Frontotemporal dementia-frontotemporal lobar degeneration with TDP-43-positive inclusions (FTLD-TDP); Intrinsically-disordered proteins; Prion-like domain; TDP-43; FUS; Circular dichroism spectroscopy; Electron microscopy; Fluorescence spectroscopy; NMR spectroscopy. 


\begin{abstract}
The mechanism of the self-assembly and fibrillization of the prion-like domains lies at the heart of their physiology and pathology. Here with the same methods previously established, we aimed to further decipher the mechanism by characterizing two prion-like sequences with the electrostatic properties very different from that of the full-length TDP-43 prion-like domain with a very basic pI value: namely the $C$-half of the TDP-43 prion-like domain only abundant in Gly, Ser, Asn and GIn with a pI of $\sim 6.3$, and the FUS prion-like domain enriched with Gly, Ser, Gln and Tyr with a pI of $\sim 3.5$. Interestingly, the C-half with the TDP-43 unique hydrophobic region removed is no longer able to form insoluble aggregates/fibrils but still capable of self-assembling into the reversible hydrogel with cross- $\beta$ structures, despite being much slower than the fulllength. On the other hand, the FUS prion-like domain rapidly self-assembles into the reversible hydrogel with cross- $\beta$ fibrillar structures in $1 \mathrm{mM}$ phosphate buffer at $\mathrm{pH} 6.8$ but its self-assembly becomes very slow in $50 \mathrm{mM}$ MES buffer at $\mathrm{pH}$ 5.5. Our study reveals that despite having completely different electrostatic properties, the full-length and C-half of the TDP-43 prion-like domain, as well as FUS prion-like domain all have the similar pH-dependence in self-assembly as we previously reported (Lim et al., [2016] PLOS Biol 14:e1002338). This unambiguously indicates that the self-assembly of the prion-like domains is not generally governed by the electrostatic interaction. Rather, their self-assembly and fibrillization are specified by the sequences despite being highly polar and degenerative. Furthermore, our study provides the first evidence that the formation of reversible hydrogel with cross- $\beta$ structures is separable from fibrillization of the prion-like domain. Finally, our results also successfully reconcile the previous discrepancy about the conformation and mechanism of the self-assembly of the FUS prion-like domain.
\end{abstract}




\section{Introduction}

Prions are characteristic of their amazing capacity of adopting many distinct conformations, some of which are infectious [1-5]. While the mammalian prion protein $(\operatorname{PrP})$ causes devastating and infectious neurodegenerative diseases, yeast prions such as Sup35 have been revealed to confer selective advantages [2-4]. Yeast prions are characterized by low complexity (LC) sequences enriched in polar and uncharged amino acids such as Gln, Asn, Ser, Gly and Tyr. Recently 240 genes out of $\sim 20,000$ human protein-coding genes $(\sim 1.2 \%)$ were shown to contain at least a domain compositionally similar to yeast prions, thus termed prion-like domains [4]. Remarkably, human proteins containing the prion-like domains are highly overrepresented by those critically interacting with RNA and DNA through self-assembling into physiological and reversible droplet/hydrogel, which may be exaggerated into pathological and irreversible aggregates/fibrils characteristic of a variety of neurodegenerative diseases [3-10]. Therefore decoding molecular mechanisms underlying the self-assembly, aggregation and fibrillization of the prion-like domains lies at the heart of their physiology and pathology; and may offer rationales to further develop therapeutic strategies for the related diseases.

Previously, as facilitated by our previous discovery that protein aggregation can be significantly minimized by reducing salt concentrations [10-17], we showed that although the TDP-43 prion-like domain is intrinsically disordered, it has the capacity to spontaneously self-assemble into dynamic and cross- $\beta$ oligomers while ALS-causing point mutations are sufficient to exaggerate the formation of the amyloid-fibers [10]. Very interestingly, we observed that the self-assembly of the TDP-43 prion-like domain is highly $\mathrm{pH}$-dependent: it showed no significant oligomerization at acidic $\mathrm{pH}$ but started to self-assemble at neutral $\mathrm{pH}$. To rationalize this, we proposed [10] that at acidic $\mathrm{pH}$, the majority of the side chain atoms of Asn, Gln and Ser are involved in forming hydrogen bonds with the backbone atoms as 
extensively observed in both well-folded proteins as well as disordered short peptides [18-20]. However, the self-assembly will be initiated by the liberation of the Asn, Gln and Ser side chains at neutral $\mathrm{pH}$ to form inter-molecular "hydrogen bonds/steric zippers" as well established [3, 6, 21, 22]. As such, despite being highly sequence-degenerative and intrinsically disordered, the prion-like domains, such as human TDP-43, appear to represent a subgroup of the intrinsically disordered proteins that can achieve extremely high specificity in the self-assembly on the basis of the global networks constituted by sequence-dependent intramolecular hydrogen-bonding/interactions [10]. Very recently, however, it was proposed in a formal comment that the $\mathrm{pH}$-dependent assembly of the TDP-43 prion-like domain we observed [10] can be simply interpreted as "Electrostatic Repulsion Governs TDP-43 Cterminal Domain Aggregation” [23].

To adequately clarify this issue, here we present the new experimental results on the C-half of the TDP-43 prion-like domain over residues 342-414 (Fig 1A) and the FUS prionlike domain over residues 1-165 (Fig 1B). Based on our previous NMR characterization [10], the TDP-43 prion-like domain radically differs from other prion-like domains in having a unique hydrophobic region over Met307-Gln344, which transforms into a well-folded $\Omega$ loop-helix motif upon being embedded in membrane environments. So here we focused only on the 73-residue C-half of the TDP-43 prion-like domain with a $\mathrm{pI}$ of $\sim 6.3$, which is mostly enriched in Asn, Gln, Ser and Gly (Fig 1C). Furthermore, we also studied the FUS prion-like domain with a $\mathrm{pI}$ of $\sim 3.5$, which has much higher contents of Gln and Tyr than those of the C-half of the TDP-43 prion-like domain (Fig 1D). 

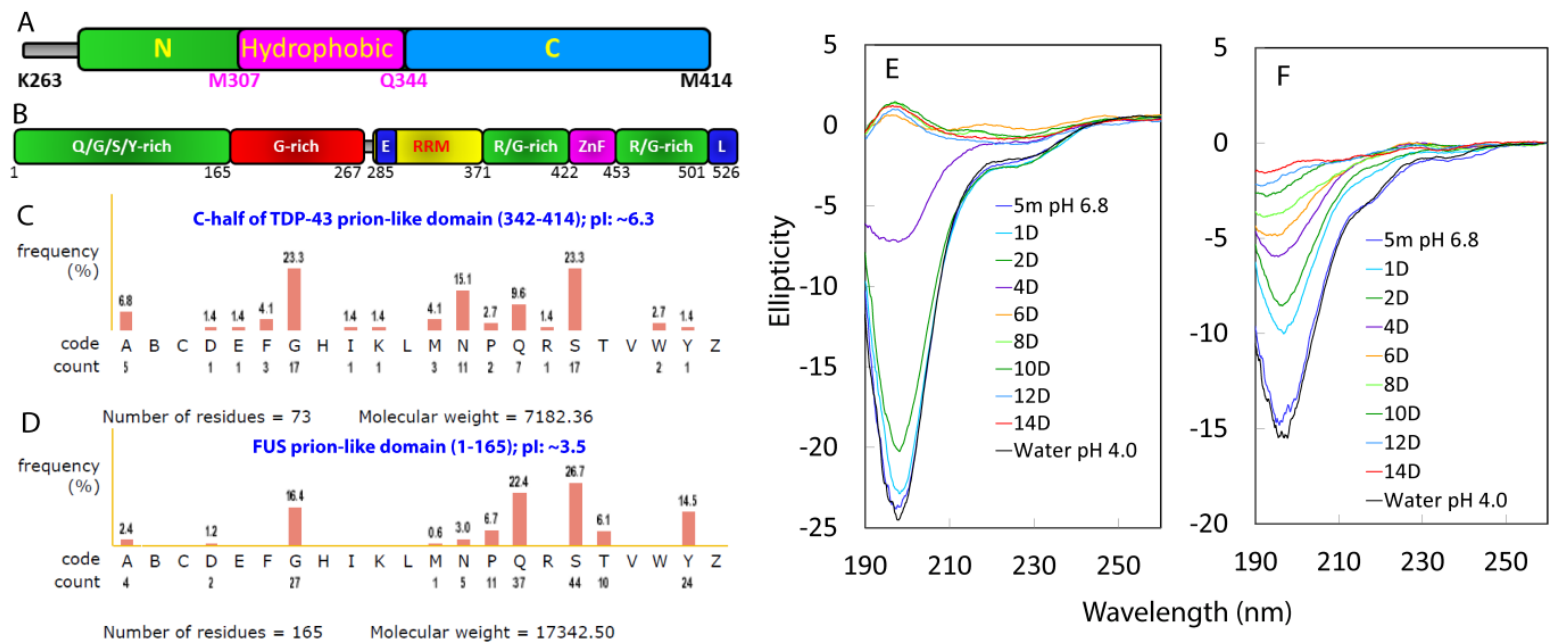

Fig 1. Domain organizations and bioinformatics analysis.

Domain organizations of the prion-like domain of TDP-43 (A); and FUS protein (B). Amino acid compositions of the C-half of the TDP-43 prion-like domain (C); and FUS prion-like domain (D). Far-UV CD spectra of the C-half (E); and FUS prion-like domain (F) at a protein concentration of $40 \mu \mathrm{M}$ in Milli-Q water ( $\mathrm{pH} 4.0)$ as well as at different time points of the incubation in $1 \mathrm{mM}$ phosphate buffer ( $\mathrm{pH} 6.8$ ). 


\section{Results}

\section{The self-assembly of the C-half.}

In Milli-Q water ( $\mathrm{pH} 4.0$ ), the $\mathrm{C}$-half has a predominantly disordered conformation very similar to that of the full-length [10], as evident from its CD (Fig 1E). Furthermore, the C-half residues adopt the solution conformation very similar to that of the corresponding region of the full-length TDP-43 prion-like domain, as judged from the fact that most of its HSQC peaks are superimposable to those of the corresponding residues of the full-length (Fig 2A). Like the full-length, it showed no detectable self-assembly or aggregation for several months at high protein concentrations $(\sim 300 \mu \mathrm{M})$ in Milli-Q water $(\mathrm{pH} 4.0)$. However, upon diluting into $1 \mathrm{mM}$ phosphate buffer ( $\mathrm{pH} 6.8), ~ 60 \%$ HSQC peaks disappeared, particularly over the N-terminal Gln-rich region (Fig 2B-2D). On the other hand, its 1D NMR peaks for the methyl protons at $\mathrm{pH} 6.8$ remained very similar to those in Milli-Q water (Fig 2E), suggesting that the disappearance of the HSQC peaks is mostly due to the rapid exchange of these exposed backbone amide protons with the bulk water at $\mathrm{pH} 6.8$.
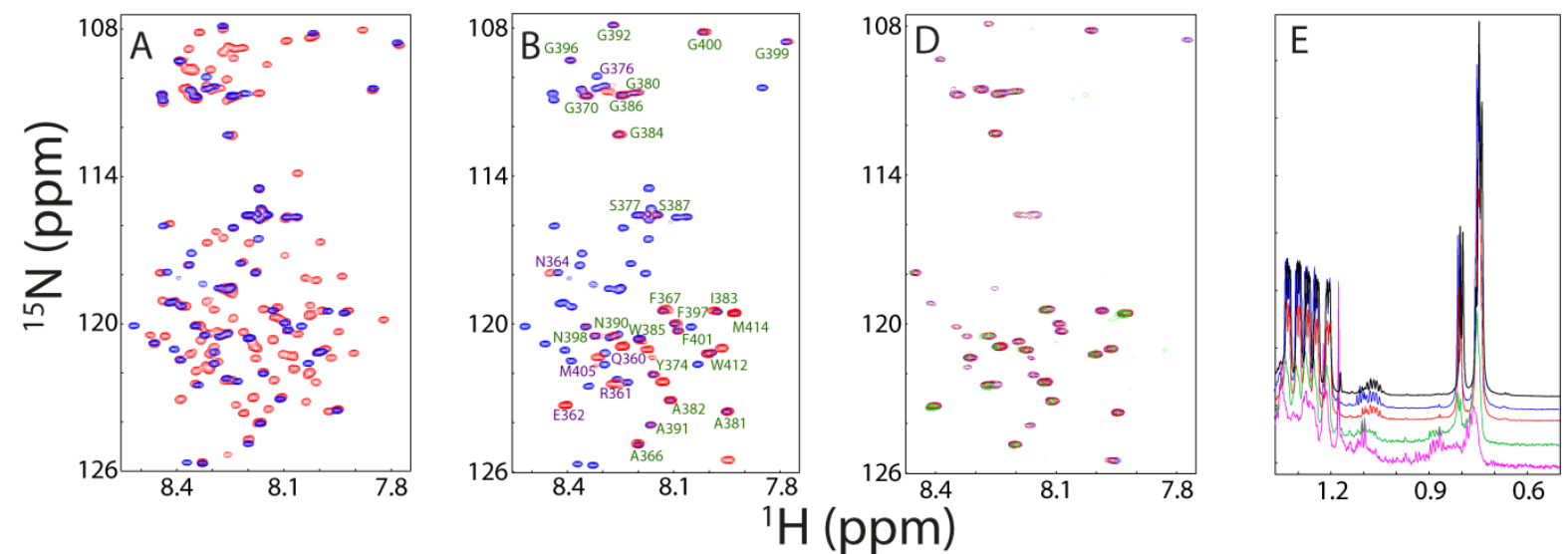

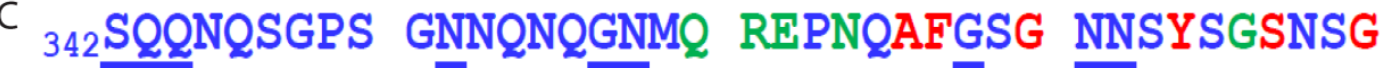 AAIGWGSASN AGSGSGFNGG FGSSMDSKSS GWGM $_{414}$}

Fig 2. NMR characterization of the C-half.

(A) Superimposition of HSQC spectra of the full-length TDP-43 prion-like domain (red) and C-half (blue) at a protein concentration of $40 \mu \mathrm{M}$ in Milli-Q water at $\mathrm{pH}$ 4.0. (B) Superimposition of HSQC spectra of the C-half in Milli-Q water at $\mathrm{pH} 4.0$ (blue) and that 
immediately diluted into $1 \mathrm{mM}$ phosphate buffer at $\mathrm{pH} 6.8$ (red) at a protein concentration of $40 \mu \mathrm{M}$. (C) The C-half sequence with the residues unassigned underlined; those retained and superimposable to those in Milli-Q water at $\mathrm{pH} 4.0$ (red), retained but significantly shifted (green) upon immediately dilution into $1 \mathrm{mM}$ phosphate buffer at $\mathrm{pH}$ 6.8. (D) Superimposition of HSQC spectra of the C-half at a protein concentration of $40 \mu \mathrm{M}$ immediately diluted into $1 \mathrm{mM}$ phosphate buffer at $\mathrm{pH} 6.8$ (blue); at day 6 (red); day 10 (green). (E) One-dimensional NMR spectra over $0.5-1.35 \mathrm{ppm}$ at a protein concentration of $40 \mu \mathrm{M}$ acquired in Milli-Q water at $\mathrm{pH} 4.0$ (black); immediately diluted into $1 \mathrm{mM}$ phosphate buffer at pH 6.8 (blue), 6 day (red), 10 day (green) and 14 day (purple).

Like the full-length, at $\mathrm{pH} 6.8$ the C-half was still capable of gradually selfassembling into oligomers rich in $\beta$-structure as monitored by $\mathrm{CD}$, fluorescence and EM. As seen in Fig 1E, in the first 4 days, the absolute values of the CD signal at $\sim 198 \mathrm{~nm}$ continuously reduced, indicating a spontaneous and gradual self-assembly. A transition occurred from 4 to 6 day. After 6 day, the CD spectra remained largely unchanged, which are typical of the soluble cross- $\beta$ oligomers such as formed by the wild type TDP-43 prion-like domain [10]. Consistent with the CD results, both HSQC (Fig 2D) and 1D (Fig 2E) spectra at day 6 remained highly similar, only with a slight reduction of peak intensity, as compared to those collected at $15 \mathrm{~min}$ after dilution into $1 \mathrm{mM}$ phosphate buffer at $\mathrm{pH}$ 6.8. However, in 10 day, the intensity of the HSQC and 1D peaks became significantly reduced and at day 14, almost all HSQC peaks became too broad to be detected. Furthermore, after 10 days, the sample became dynamic hydrogel at $4{ }^{\circ} \mathrm{C}$ but could be converted back to liquid at room temperature or/and by shaking as previously observed on the FUS [6] and full-length TDP-43 prion-like domains [10].

Interestingly, the time-lapse change of the intrinsic UV fluorescence revealed a multiphase process of the self-assembly of the C-half which contains two Trp residues: Trp385 and Trp412 (Fig 2C). As seen in the upper panel of Fig 3A, similar to what was observed on the full-length in Milli-Q water at $\mathrm{pH} 4.0$ [10], the C-half has an intrinsic UV fluorescence spectrum with the emission maximum of $352 \mathrm{~nm}$, which implies that both Trp residues are 
highly exposed as in the full-length. Immediately after dilution into the $1 \mathrm{mM}$ phosphate buffer at $\mathrm{pH}$ 6.8, a slight increase of the intensity was observed. However, within the first 2 days of the incubation, the intensity reduced and the emission maximum became largely blueshifted to $\sim 332 \mathrm{~nm}$. Amazingly, from day 4 to day 6 , the spectra suddenly have two emission maxima: one at $\sim 335 \mathrm{~nm}$ and another at $\sim 385 \mathrm{~nm}$. This implies that in this period of the selfassembly, two Trp residues may experience different chemical environments. After day 8, the intensity further reduced and only one emission maximum was observed at $\sim 324 \mathrm{~nm}$, suggesting that both Trp residues became highly buried.
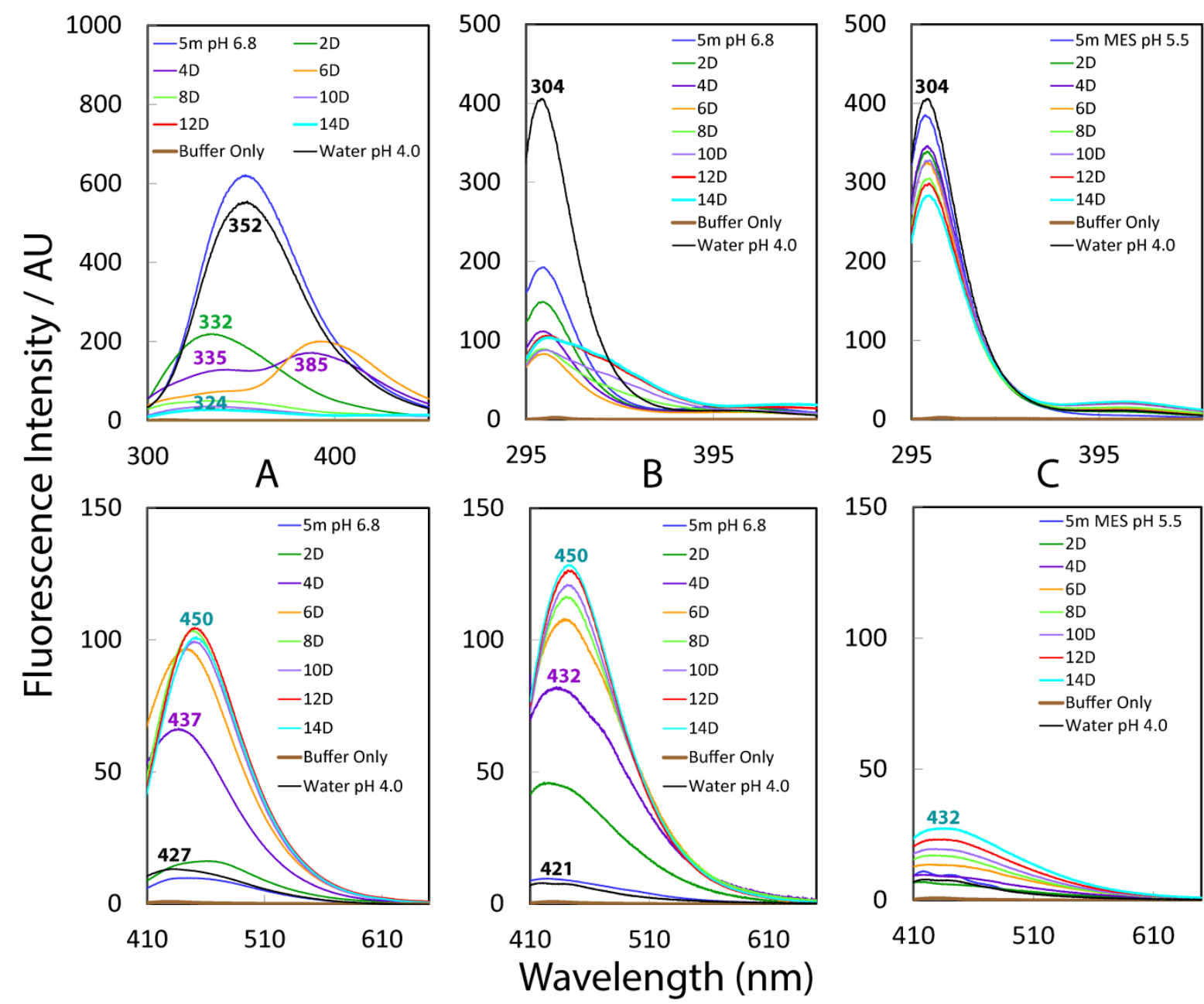

Fig 3. Fluorescence characterization. Emission spectra of the intrinsic UV (upper panel) and visible (lower panel) fluorescence at different time points of the incubation at a protein concentration of $40 \mu \mathrm{M}$ respectively for the C-half of the TDP-43 prion-like domain in $1 \mathrm{mM}$ phosphate buffer at $\mathrm{pH} 6.8$ (A), FUS prion-like domain in $1 \mathrm{mM}$ phosphate buffer at $\mathrm{pH} 6.8$ (B) and FUS prion-like domain in $50 \mathrm{mM}$ MES buffer at $\mathrm{pH} 5.5$ (C). 
Recently, an intrinsic visible fluorescence has been established to be a signature for the cross- $\beta$ structures, which is independent of the presence of aromatic residues but have its origin in the formation of aligned hydrogen bond networks involved in the backbone $\mathrm{C}=\mathrm{O}$ and $\mathrm{N}-\mathrm{H}$ atom groups of peptide bonds $[10,24,25]$. Like the full-length, the C-half samples both in Milli-Q water at $\mathrm{pH} 4.0$ and immediately diluted in $1 \mathrm{mM}$ phosphate buffer at $\mathrm{pH} 6.8$ already have the intrinsic visible fluorescence but its intensity (12.8) is lower than that (25.1) of the full-length, while the emission maximum $(\sim 432 \mathrm{~nm})$ is also less red-shifted than that of the full-length $(\sim 446 \mathrm{~nm})$ (lower panel of Fig 3A). Nevertheless, with the time lapse, the intensity continuously increased and consequently after 12 days, it reached the highest (104.3) and the emission maximum red-shifted to $\sim 450 \mathrm{~nm}$. By contrast, under the same conditions, only after 2 days, the intensity of the full-length reached the highest $(\sim 62.5)$ with the emission maximum red-shifted to $\sim 450 \mathrm{~nm}$. This indicate that: 1) the self-assembly of the Chalf is much slower than that of the full-length; 2) the C-half could still form reversible hydrogel even with the higher content of cross- $\beta$ structures.

Unexpectedly, however, very different from the full-length, as imaged by EM, the Chalf failed to form any insoluble aggregates/fibrillar structures even after 2 weeks of the incubation (Fig 4A). By contrast, under the exact same conditions, both 1D and HSQC peaks of the full-length became very broad after $24 \mathrm{hr}$, and further incubation led to formation of insoluble fibrils [10]. The fact that the C-half with a $\mathrm{pI}$ of $\sim 6.3$ self-assembled into the cross- $\beta$ hydrogel much more slowly than the full-length with a pI of 10.9, clearly indicates that the self-assembly of the TDP-43 prion-like domain is not simply governed by electrostatic interaction. 


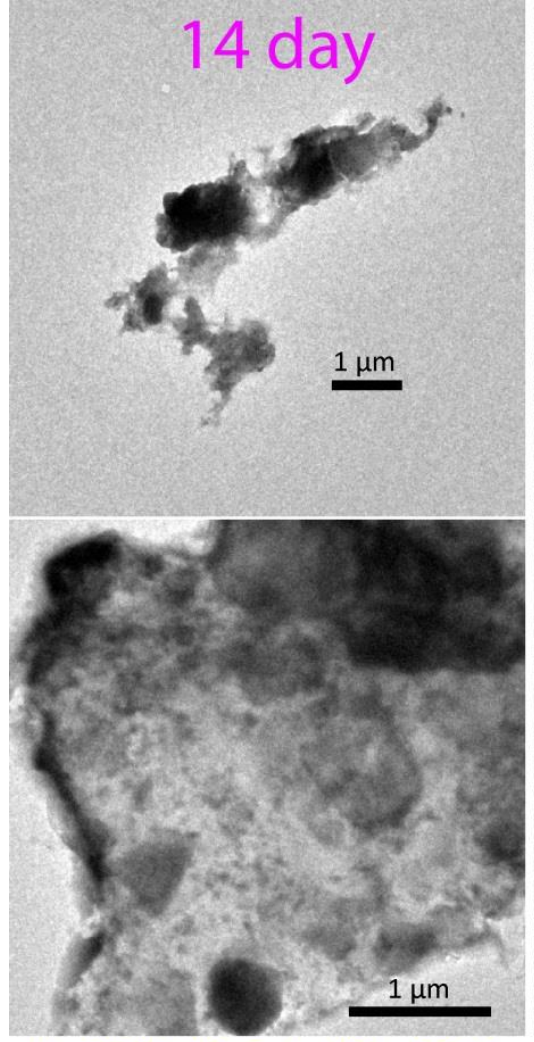

\section{A: TDP-43 C-half in PO4 buffer $\mathrm{pH} 6.8$}

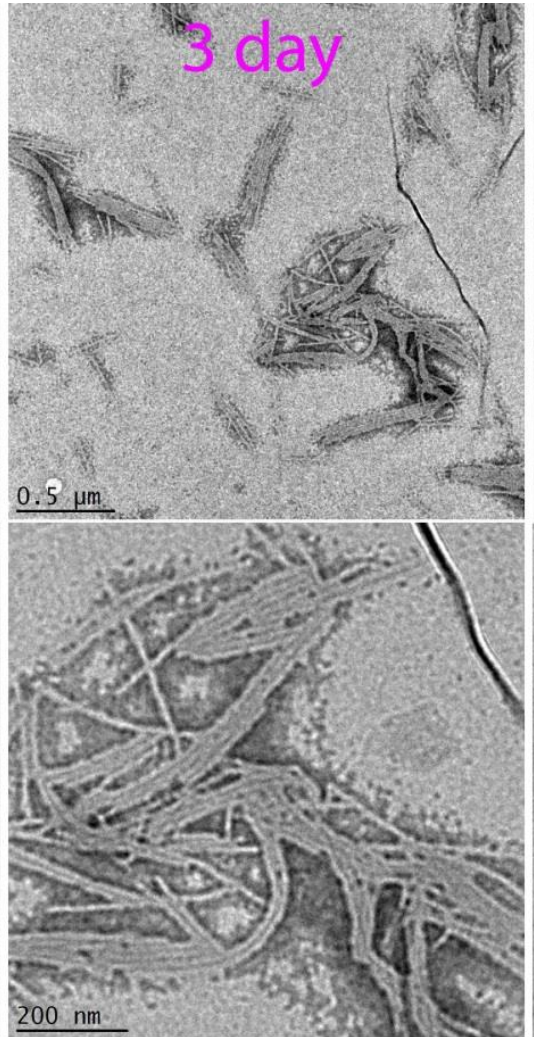

B: FUS in $\mathrm{PO} 4$ buffer pH 6.8

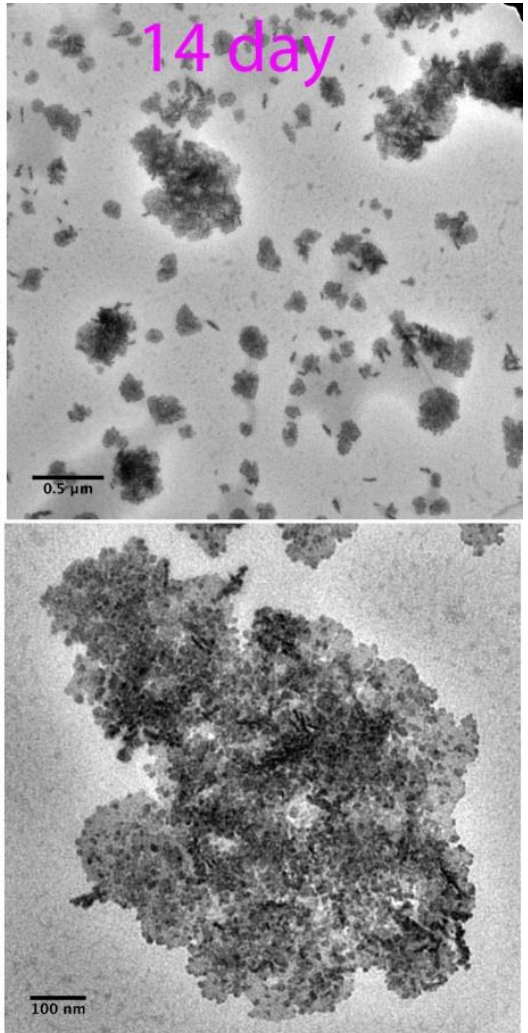

C: FUS in MES buffer $\mathrm{pH} 5.5$

Fig 4. EM imaging.

EM images of the samples of the C-half incubated for 14 days in $1 \mathrm{mM}$ phosphate buffer at $\mathrm{pH} 6.8$ (A); of FUS prion-like domain incubated for 3 days in $1 \mathrm{mM}$ phosphate buffer at $\mathrm{pH}$ 6.8 (B); and of FUS prion-like domain incubated for 14 days in $50 \mathrm{mM}$ MES buffer at $\mathrm{pH} 5.5$ (C).

\section{The self-assembly and fibrillization of the FUS prion-like domain.}

Intriguingly, despite having an acidic pI value, the FUS prion-like domain also showed no detectable self-assembly at high concentrations $(\sim 400 \mu \mathrm{M})$ in Milli-Q water $(\mathrm{pH}$ 4.0). It has a highly disordered conformation in Milli-Q water ( $\mathrm{pH} 4.0)$, as indicated by its CD spectrum with the maximum negative signal at $~ 196 \mathrm{~nm}$ but no positive signal (Fig 1F). Furthermore, we have acquired one-dimensional and two-dimensional NMR HSQC spectra for the FUS prion-like domain at a protein concentration of $40 \mu \mathrm{M}$ in Milli-Q water at $\mathrm{pH} 4.0$, $1 \mathrm{mM}$ phosphate buffer at $\mathrm{pH} 5.0$ and 6.8 respectively, as well as in $50 \mathrm{mM}$ MES (Nmorpholinoethanesulfonic acid) buffer at pH 5.5 respectively (Fig 5A-5D). In Milli-Q water 
at $\mathrm{pH}$ 4.0, the FUS prion-like domain has a narrowly-dispersed HSQC spectrum with only the ${ }^{1} \mathrm{H}$ dispersion of $0.95 \mathrm{ppm}$ and ${ }^{15} \mathrm{~N}$ dispersion of $17.3 \mathrm{ppm}$ (Fig 5A); and has no very up-field peaks with negative chemical shifts in its 1D spectrum (Fig 5B). This indicates that the FUS prion-like domain has no tight tertiary packing. However, very different from what we observed on the TDP-43 prion-like domain [10], even in $1 \mathrm{mM}$ phosphate buffer at $\mathrm{pH}$ 5.0, a portion of HSQC peaks completely disappeared and many HSQC peaks shifted (Fig 5A), clearly indicating that these amide protons might be highly exposed to the bulk solvent. On the other hand, its $1 \mathrm{D}$ spectrum over 1-2 ppm at pH 5.0 is still very similar to that in Milli-Q water at $\mathrm{pH} 4.0$ (Fig 5B), indicating that no significantly self-assembly occurred shortly at $\mathrm{pH}$ 5.0. Interestingly, the majority of its HSQC peaks at $\mathrm{pH} 4.0$ and $\mathrm{pH} 5.0$ (Fig 5A) are superimposable to those in $50 \mathrm{mM}$ MES buffer at $\mathrm{pH} 5.5$ (Fig 5C), under which the same FUS prion-like domain has been characterized to exist as a highly disordered monomer by NMR [9].

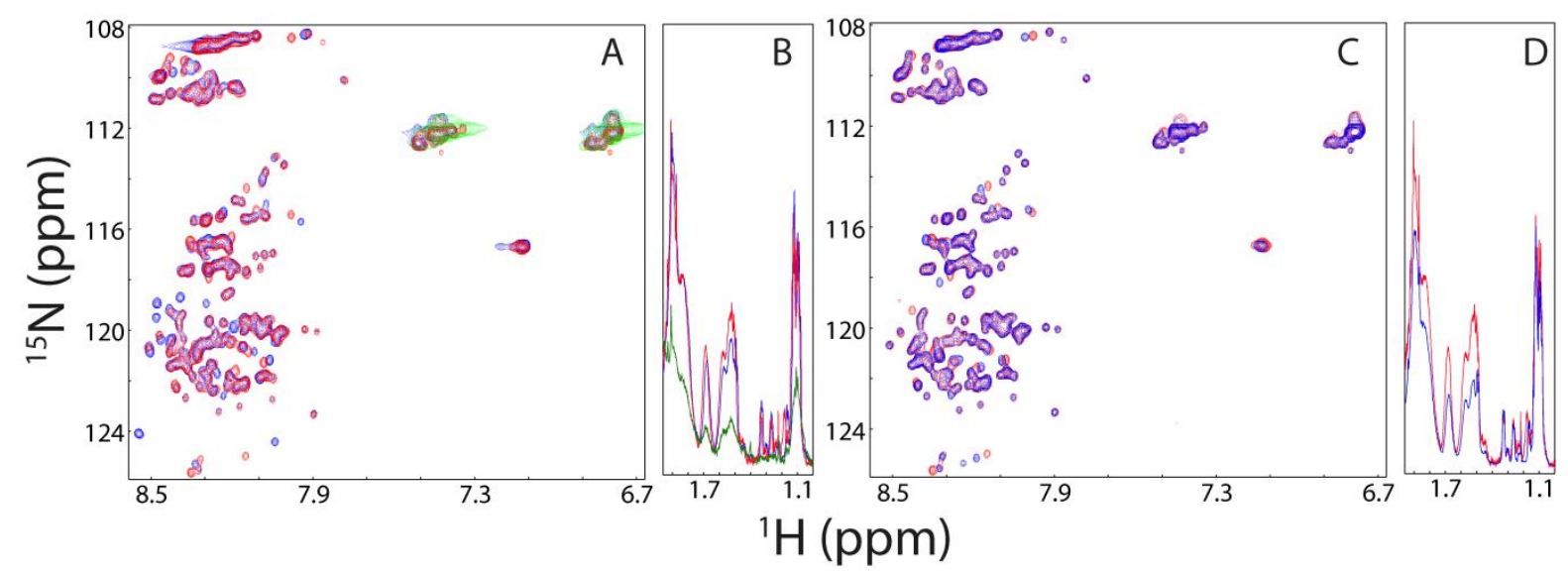

Fig 5. NMR characterization of the FUS prion-like domain.

Superimposition of HSQC (A) and 1D ${ }^{1} \mathrm{H}$ (B) NMR spectra of the FUS prion-like domain in Milli-Q water at $\mathrm{pH} 4.0$ (blue); in $1 \mathrm{mM}$ phosphate buffer at $\mathrm{pH} 5.0$ (red); and at $\mathrm{pH} 6.8$ (green). Superimposition of HSQC (C) and 1D ${ }^{1} \mathrm{H}$ (D) NMR spectra of the FUS prion-like domain in $1 \mathrm{mM}$ phosphate buffer at $\mathrm{pH} 5.0$ (blue) and in $50 \mathrm{mM}$ MES buffer at pH 5.5 (red).

However, upon diluting the FUS prion-like domain into $1 \mathrm{mM}$ phosphate buffer at $\mathrm{pH}$ 6.8, its 1D peaks became very broad (Fig 5B), while almost all backbone HSQC peaks 
disappeared (Fig 5A), mostly due to rapid exchanges of amide protons with water, or/and the broadening of peaks resulting from the rapid self-assembly into large oligomers as previously characterized [10]. This is very different from the previous results with the full-length TDP43 prion-like domains: even in $1 \mathrm{mM}$ phosphate buffer at $\mathrm{pH} 6.8$, the majority of HSQC peaks was still detectable and only became too broad to be detectable after 4 day at the same concentration of $40 \mu \mathrm{M}$. The present results clearly indicate that the FUS prion-like domain has its backbone amide protons much less protected, or/and a tendency in self-assembly much higher than TDP-43 [10].

Consistent with the above NMR observation, very large changes of the CD intensity were observed for FUS prion-like domain after 1 day (Fig 1F), suggesting its rapid selfassembly. As the FUS prion-like domain has no Trp but has 24 Tyr residues, its intrinsic UV fluorescence from Tyr has the maximum emission wavelength at $\sim 304 \mathrm{~nm}$ (Upper panel of Fig 3B). Interestingly, its fluorescence intensity significantly reduced immediately upon being diluted into $1 \mathrm{mM}$ phosphate buffer at $\mathrm{pH}$ 6.8. For the intrinsic visible fluorescence (Lower panel of Fig 3B), although FUS prion-like domain has very weak emission signal 5 min after diluting into $1 \mathrm{mM}$ phosphate buffer at $\mathrm{pH} 6.8$, it rapidly developed after 2 days with the emission maximum at $\sim 425 \mathrm{~nm}$ and intensity of 46.2. After 4 days it further developed with the emission maximum red-shifted to $\sim 432 \mathrm{~nm}$ and intensity increased to of 81.8. After 14 days it fully developed with the emission maximum red-shifted to $\sim 450 \mathrm{~nm}$ and intensity increased to of 126.5 , which is even much higher than that developed for the full-length TDP-43 prion-like domains [10]. The results reveal that at $\mathrm{pH} 6.8$, the highly disordered FUS prion-like domain could indeed self-assemble into the hydrogel with cross- $\beta$ structures. Furthermore, it forms fibrillar structures with diameter of 10-20 nm even in 3 days (Fig 4B), and no significant change of the morphology was observed even after 14 days. Our 
current results are completely consistent with the self-assembly of the FUS prion-like domain previously revealed by EM and other biophysical probes including X-ray diffraction [6].

We also prepared a sample of the FUS prion-like domain in $50 \mathrm{mM}$ MES buffer at $\mathrm{pH}$ 5.5, which was previously used to NMR characterize the same FUS prion-like domain [9]. Due to the large noise from the MES buffer, the self-assembly could not be monitored by farUV CD spectroscopy. Nevertheless, it has HSQC and 1D NMR spectra very similar to those in $1 \mathrm{mM}$ phosphate buffer at $\mathrm{pH} 5.0$ (Fig 5C and 5D). Furthermore, very small change in the intrinsic UV fluorescence was observed upon immediate dilution into $50 \mathrm{mM}$ MES buffer at pH 5.5 (Upper panel of Fig 3C). Most remarkably, unlike in $1 \mathrm{mM}$ phosphate buffer at pH 6.8 (Upper panel of Fig 3B), in $50 \mathrm{mM}$ MES buffer at $\mathrm{pH} 5.5$, only small changes in the intrinsic UV fluorescence have been detected with the incubation up to 14 days (Upper panel of Fig 3C). Consistent with the UV intrinsic fluorescence, only very small increases in the intrinsic visible fluorescence with minor red-shifts have been observed during incubation up to 14 days (Lower panel of Fig 3C). Moreover, the sample remained transparent and failed to form hydrogel even up to 14 days. Indeed, no fibrillar structure was detected by EM for the sample incubated up to 14 days (Figure 4C). 


\section{Discussion}

To further decipher the molecular determinant for the self-assembly and fibrillization of the prion-like domains, with the same methods we previously utilized [10], we characterized two prion-like sequences with electrostatic properties different from that of the full-length TDP-43 prion-like domain, which are only enriched in polar and uncharged residues Gly, Ser, Asn, Gln and Tyr characteristic of the prion-like domains [4]. The new results, together with previous one [10] reveal that despite the complete difference of their electrostatic properties, all three prion-like domains have the same $\mathrm{pH}$-dependent selfassembly: the self-assembly is significantly inhibited at acidic $\mathrm{pH}$ while it is initiated at neutral $\mathrm{pH}$ as we reported before [10]. Strikingly, the C-half with its $\mathrm{pI}$ much closer to neutral $\mathrm{pH}$ than the full-length self-assembled much slowly than the full-length TDP-43 prion-like domain. The results together clearly indicate that the self-assembly of the prion-like domains is not generally governed by the electrostatic interactions as commented [23]. Therefore, to the best of our knowledge, the pH-dependent self-assembly of the TDP-43 and FUS prionlike domains can only be rationalized by the mechanism we previously proposed [10]. This mechanism is further supported by the observation here that the backbone amide protons of the FUS prion-like domain are much more significantly perturbed by the $\mathrm{pH}$ changes, as the FUS prion-like domain contains much higher content of Gln than the TDP-43 prion-like domain (Fig 1D). It has been shown that the hydrogen bonds formed between side chain and backbone atoms of Gln are less stable than those of Asn [18-20].

Our current results show for the first time that the self-assembly into dynamic hydrogels with significant cross- $\beta$ structures and fibrillization are separable for the prion-like domains. This suggests that the prion-like sequences enriched in the polar and uncharged residues are able to spontaneously self-assemble into dynamic hydrogels with significant cross- $\beta$ structures, but whether they can further fibrillize is highly dependent on the strength 
of the interactions. For example, the full-length TDP-43 prion-like domain appears to employ the hydrophobic interaction offered by the middle region, while the FUS prion-like domain utilizes the aromatic interaction provided a large number of Tyr residues to drive further fibrillization. It is highly possible that many environmental factors may act to enhance the interaction strength, thus driving the jump from dynamic hydrogels into fibrillar structures, both of which share the same cross- $\beta$ structures.

Finally, our results also successfully reconcile the previous discrepancy about the mechanism by which the FUS prion-like domain drives the self-assembly: in one study conducted in neutral $\mathrm{pH}$, the FUS prion-like domain has been characterized to self-assemble into dynamic hydrogel with fibrillar structures [6], while in a later NMR characterization performed in $50 \mathrm{mM}$ MES buffer at $\mathrm{pH}$ 5.5, the FUS prion-like domain was shown to remain highly disordered even upon forming droplets active for binding [9]. Based on our present results, it seems that the relatively disordered state previously observed [9] represents a snapshot of the self-assembly at the early stages, while the dynamic hydrogel with fibrillar structures [6] reflects the structures formed at the late stages. Taken together, our results suggest that the formation of cross- $\beta$ structures represents a common mechanism for the selfassembly of the prion-like domains, but whether further fibrillization occurs is mediated by the intrinsic sequence-specific features as well as environmental factors. In cells, different snapshots of the self-assembly might be all utilized for implementing the biological functions by the proteins containing the prion-like domains $[6-9,26]$. 


\section{Methods}

\section{Preparation of recombinant proteins}

The DNA fragments encoding the C-half of the TDP-43 prion-like domain (342-414) and FUS prion-like domain (1-165) were amplified by PCR reactions from TDP-43 and FUS cDNA and subsequently cloned into a modified vector pET28a with 6 His residues at Cterminus as we previously used for the TDP-43 prion-like domain [10]. The expression vectors were subsequently transformed into and overexpressed in Escherichia coli BL21 (DE3) cells (Novagen). The recombinant protein of the C-half of the TDP-43 prion-like domain was found in supernatant while FUS prion-like domain in inclusion body. As a result the TDP-43 prion-like domain was purified by a $\mathrm{Ni}^{2+}$-affinity column (Novagen) under native conditions; while FUS prion-like domain was purified by a $\mathrm{Ni}^{2+}$-affinity column (Novagen) under denaturing conditions in the presence of $8 \mathrm{M}$ urea. The fractions containing the recombinant proteins were acidified by adding $10 \%$ acetic acid and subsequently purified by reverse-phase (RP) HPLC on a C4 column eluted by water-acetonitrile solvent system. The HPLC elutions containing pure recombinant proteins were lyophilized [27].

The generation of the isotope-labelled proteins for NMR studies followed a similar procedure except that the bacteria were grown in M9 medium with the addition of $\left({ }^{15} \mathrm{NH}_{4}\right)_{2} \mathrm{SO}_{4}$ for ${ }^{15} \mathrm{~N}$ labeling and $\left({ }^{15} \mathrm{NH}_{4}\right)_{2} \mathrm{SO}_{4} /\left[{ }^{13} \mathrm{C}\right]$-glucose for double labelling [27]. The purity of the recombinant proteins was checked by SDS-PAGE gels and their molecular weights were verified by a Voyager STR matrix-assisted laser desorption ionization time-offlight-mass spectrometer (Applied Biosystems). The concentration of protein samples was determined by the UV spectroscopic method in the presence of $8 \mathrm{M}$ urea. Briefly, under the denaturing condition, the extinct coefficient at $280 \mathrm{~nm}$ of a protein can be calculated by adding up the contribution of Trp, Tyr and Cys residues. 


\section{CD and NMR experiments}

All circular dichroism (CD) experiments were performed on a Jasco J-810 spectropolarimeter equipped with a thermal controller using 1-mm path length cuvettes. Data from five independent scans were added and averaged. CD samples were prepared by diluting the concentrated samples $(\sim 300 \mu \mathrm{M})$ dissolved in Milli-Q water $(\mathrm{pH} 4.0)$ into different buffers respectively. All NMR experiments were acquired on an $800 \mathrm{MHz}$ Bruker Avance spectrometer equipped with pulse field gradient units as described previously $[27,28]$.

\section{Fluorescence spectral measurements}

All fluorescence spectra were measured at $25{ }^{\circ} \mathrm{C}$ with a RF-5301 PC spectrophotometer (Shimadzu, Japan) as previously established [10] at different time points of the incubations at a protein concentration of $40 \mu \mathrm{M}$ in different buffers. The rectangular fluorescence quartz cuvette has the pathlength dimension of $10 \times 10 \mathrm{~mm}$ and the general settings are: PMT at low sensitivity and scan speed of medium speed $(200 \mathrm{~nm} / \mathrm{min})$. For the intrinsic UV fluorescence, the emission spectra were measured with the excitation wavelength at $280 \mathrm{~nm}$ and slit widths: excitation at $5 \mathrm{~nm}$ and emission at $10 \mathrm{~nm}$. For the intrinsic visible fluorescence, the emission spectra were measured with the excitation wavelength at $375 \mathrm{~nm}$ and slit widths: excitation at $20 \mathrm{~nm}$ and emission at $10 \mathrm{~nm}$.

\section{Electron microscopy imaging}

Incubation samples at a protein concentration of $40 \mu \mathrm{M}$ were imaged at different time points by a TEM microscope (Jeol Jem 2010f Hrtem, Japan) operating at an accelerating voltage of $200 \mathrm{kV}$. For EM imaging, a $5 \mu \mathrm{l}$ aliquot of the incubation or aggregate solutions was placed onto the $\mathrm{Cu}$ grids (coated with carbon film; 150 mesh; 3mm in diameter) and 
negatively stained with $5 \mu$ of $2 \%$ neutral, phosphotungstic acid (PTA). This aliquot was allowed to settle on $\mathrm{Cu}$ grid for $30 \mathrm{sec}$ before the excess fluid was drained away. The $\mathrm{Cu}$ grid was later air-dried for another 15 mins before being imaged. 


\section{References}

1. Prusiner S.B. (1998) Prions. Proc. Natl. Acad. Sci. USA. 95, 13363-13383.

2. Lindquist S. (1997) Mad cows meet psi-chotic yeast: the expansion of the prion hypothesis. Cell 89, 495-498.

3. Michelitsch M.D, Weissman JS. (2000) A census of glutamine/asparagine-rich regions: implications for their conserved function and the prediction of novel prions. Proc. Natl. Acad. Sci. USA. 97, 11910-11915.

4. March ZM, King OD, Shorter J. (2016) Prion-like domains as epigenetic Regulators, scaffolds for subcellular organization, and drivers of neurodegenerative disease. Brain Res. Mar 17. pii: S0006-8993(16)30096-8.

5. Polymenidou M. Cleveland D.W. (2011) The seeds of neurodegeneration: prion-like spreading in ALS. Cell 147, 498-508.

6. Kato M, Han TW, Xie S, Shi K, Du X, Wu LC, et al. (2012) Cell-free formation of RNA granules: low complexity sequence domains form dynamic fibers within hydrogels. Cell. 149, 753-767.

7. Schwartz JC, Wang X, Podell ER, Cech TR. (2013) RNA seeds higher-order assembly of FUS protein. Cell Rep. 5, 918-925.

8. Zhang H, Elbaum-Garfinkle S, Langdon EM, Taylor N, Occhipinti P, Bridges AA, Brangwynne CP, Gladfelter AS. (2015) RNA Controls PolyQ Protein Phase Transitions. Mol Cell. 60, 220-30.

9. Burke KA, Janke AM, Rhine CL, Fawzi NL. (2015) Residue-by-Residue View of In Vitro FUS Granules that Bind the C-Terminal Domain of RNA Polymerase II. Mol Cell. 60, 23141. 
10. Lim L, Wei Y, Lu Y, Song J (2016) ALS-Causing Mutations Significantly Perturb the Self-Assembly and Interaction with Nucleic Acid of the Intrinsically Disordered Prion-Like Domain of TDP-43. PLoS Biol 14, e1002338.

11. Song J. (2009) Insight into "insoluble proteins" with pure water. FEBS Lett. 583, 953959.

12. Song J. (2013) Why do proteins aggregate? "Intrinsically insoluble proteins" and "dark mediators" revealed by studies on "insoluble proteins" solubilized in pure water. F1000Research. 2, 94.

13. Shi J, Lua S, Tong JS, Song J. (2010) Elimination of the native structure and solubility of the hVAPB MSP domain by the Pro56Ser mutation that causes amyotrophic lateral sclerosis. Biochemistry. 49, 3887-97.

14. Qin H, Lim L, Wei Y, Gupta G, Song J. (2013) Resolving the paradox for protein aggregation diseases: NMR structure and dynamics of the membrane-embedded P56S-MSP causing ALS imply a common mechanism for aggregation-prone proteins to attack membranes. Version 2. F1000Res. 2013 Oct 21 [revised 2014 Jul 22];2:221. doi: 10.12688/f1000research.2-221.v2. eCollection 2013.

15. Lim L, Lee X, Song J. (2014) Mechanism for transforming cytosolic SOD1 into integral membrane proteins of organelles by ALS-causing mutations. Biochim Biophys Acta. 1848, $1-7$.

16. Lim L, Song J. (2016) SALS-linked WT-SOD1 adopts a highly similar helical conformation as FALS-causing L126Z-SOD1 in a membrane environment. Biochim Biophys Acta. 1858, 2223-30.

17. Qin H, Lim L, Wei Y, Song J. (2014) TDP-43 N terminus encodes a novel ubiquitin-like fold and its unfolded form in equilibrium that can be shifted by binding to ssDNA. Proc Natl Acad Sci U S A. 111, 18619-18624. 
18. Eswar N, Ramakrishnan C. (2000) Deterministic features of side-chain main-chain hydrogen bonds in globular protein structures. Protein Eng. 13, 227-238.

19. Vasudev PG, Banerjee M, Ramakrishnan C, Balaram P. (2012) Asparagine and glutamine differ in their propensities to form specific side chain-backbone hydrogen bonded motifs in proteins. Proteins. 80, 991-1002.

20. Song B, Kibler P, Malde A, Kodukula K, Galande AK. (2010) Design of short linear peptides that show hydrogen bonding constraints in water. J Am Chem Soc. 132, 4508-4509. 21. Perutz MF, Johnson T, Suzuki M, Finch JT. (1994) Glutamine repeats as polar zippers: their possible role in inherited neurodegenerative diseases. Proc. Natl. Acad. Sci.USA. 91, $5355-5358$.

22. Eisenberg D, Jucker M. (2012) The amyloid state of proteins in human diseases. Cell. 148, 1188-203.

23. Mompeán M, Chakrabartty A, Buratti E, Laurents DV. (2016) Electrostatic Repulsion Governs TDP-43 C-terminal Domain Aggregation. PLoS Biol. 14, e1002447.

24. Shukla A, Mukherjee S, Sharma S, Agrawal V, Radha Kishan KV, Guptasarma P. (2004) A novel UV laserinduced visible blue radiation from protein crystals and aggregates: scattering artifacts or fluorescence transitions of peptide electrons delocalized through hydrogen bonding? Arch Biochem Biophys. 428, 144-153.

25. Chan FT, Kaminski Schierle GS, Kumita JR, Bertoncini CW, Dobson CM, Kaminski CF. (2013) Protein amyloids develop an intrinsic fluorescence signature during aggregation. Analyst. 138, 2156-2162.

26. Xiang S, Kato M, Wu LC, Lin Y, Ding M, Zhang Y, Yu Y, McKnight SL. (2015) The LC Domain of hnRNPA2 Adopts Similar Conformations in Hydrogel Polymers, Liquid-like Droplets, and Nuclei. Cell. 163, 829-39. 
27. Lamberto I, Qin H, Noberini R, Premkumar L, Bourgin C, Riedl SJ, Song J, Pasquale EB. (2012) Distinctive binding of three antagonistic peptides to the ephrin-binding pocket of the EphA4 receptor. Biochem J. 445, 47-56.

28. Qin H, Lim LZ, Song J. (2015) Dynamic principle for designing antagonistic/agonistic molecules for EphA4 receptor, the only known ALS modifier. ACS Chem Biol. 10, 372-8. 


\section{Author Contributions}

Conceived and designed the experiments: JXS; Performed the experiments: YML LZL YMT LW. Analyzed the data: JXS YML LZL YMT. Wrote the paper: JXS.

\section{Acknowledgement}

This study is supported by Ministry of Education of Singapore (MOE) Tier 2 Grants 2011-

T2-1-096 and MOE2015-T2-1-111 to Jianxing Song. The funders had no role in study design, data collection and analysis, decision to publish, or preparation of the manuscript. 